\title{
Near-infrared intersubband transitions in InGaAs-AIAs-InAIAs double quantum wells
}

\author{
M. P. Semtsiv, ${ }^{\text {a) }}$ M. Ziegler, and W. T. Masselink \\ Department of Physics, Humboldt-Universität zu Berlin, Newtonstrasse 15, D-12489 Berlin, Germany \\ N. Georgiev, T. Dekorsy, and M. Helm \\ Institute of Ion Beam Physics and Materials Research, Forschungszentrum Rossendorf, \\ P.O. Box 510119, D-01314 Dresden, Germany
}

\begin{abstract}
Intersubband optical transitions at short wavelengths in strain-compensated $\operatorname{In}_{0.70} \mathrm{Ga}_{0.30} \mathrm{As}$ - $\mathrm{AlAs}$ double quantum wells are investigated by means of mid-infrared absorption. Trade-offs between achieving a high transition energy and a large oscillator strength of the two highest-energy intersubband transitions using our strain-compensation approach are analyzed as a function of the widths of the two wells. Two design strategies leading to relatively strong intersubband optical transitions at $800 \mathrm{meV}, 1.55 \mu \mathrm{m}$, are described and the corresponding structures grown using gas-source molecular-beam epitaxy on (001)InP are investigated. The strongest intersubband transitions obtained experimentally are generally between 300 and $600 \mathrm{meV}, 2-4 \mu \mathrm{m}$. Significant oscillator strength, however, also extends out to $800 \mathrm{meV}, 1.55 \mu \mathrm{m}$.
\end{abstract}

\section{INTRODUCTION}

Optical devices based on intersubband transitions are especially well suited for many applications in the mid-infrared to far infrared spectral regions due to their high degree of flexibility in design and operational wavelength and their intrinsically ultrashort transition times. Examples include intersubband lasers modulated up to $10 \mathrm{GHz},{ }^{1,2}$ ultra fast intersubband photodetectors, ${ }^{3-5}$ and optical switches. ${ }^{6}$ One attractive application area in ultrafast optoelectronic devices is optical communication. Intersubband transitions at $1.55 \mu \mathrm{m}$, however require very large conduction-band offset and were only recently demonstrated in the $\mathrm{ZnSe}-\mathrm{BeTe}{ }^{7,8}$ GaN-AlN, ${ }^{9,10}$ InGaAs-AlAsSb, ${ }^{6,11}$ and the InGaAsN-AlGaAs ${ }^{12}$ material systems.

The ability to achieve $1.55-\mu \mathrm{m}$ intersubband transitions in the InGaAs-InAlAs/InP material system could offer advantages over other materials systems. Because InGaAsAlAs is a strain-compensated extension of the technologically mature $\mathrm{In}_{0.53} \mathrm{Ga}_{0.47} \mathrm{As}-\mathrm{In}_{0.52} \mathrm{Al}_{0.48} \mathrm{As}$ materials system, it is being actively developed for high-performance intersubband devices (both lasers and detectors) operating in the mid-infrared spectrum. ${ }^{13-16}$ In particular, progress toward short wavelengths using InGaAs-AlAs-InAlAs straincompensated heterostructures with the average lattice constant that of InP has been reported by several groups. ${ }^{13,14,16-18}$ Smet et al. ${ }^{18}$ have reported $1.55-\mu \mathrm{m}$ intersubband transitions for three-monolayer (ML) -thick InGaAs quantum well $(\mathrm{QW})$ with AlAs barriers. This result, however, is difficult to reproduce. Georgiev et al. ${ }^{14}$ have demonstrated intersubband transitions as short as $1.72 \mu \mathrm{m}$ for six-ML thick $\operatorname{In}_{0.70} \mathrm{Ga}_{0.30} \mathrm{As}$ single QWs with composite $\mathrm{In}_{0.55} \mathrm{Al}_{0.45} \mathrm{As}-\mathrm{AlAs}$ barriers, consistent with the results of

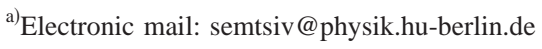

Ref. 17. In the case of single QWs, however, the use of well widths narrower than six ML results only in a decrease in absorption amplitude and not in shorter-wavelength absorption. An important issue in such structures is the strain compensation because the local tensile strain of AlAs barriers is difficult to compensate when the compressively strained wells are too thin.

In this paper we describe an approach for engineering short-wavelength intersubband transitions in coupled $\mathrm{In}_{0.70} \mathrm{Ga}_{0.30} \mathrm{As}-\mathrm{AlAs}-\mathrm{In}_{0.55} \mathrm{Al}_{0.45} \mathrm{As}$ double quantum wells (DQWs). One significant advantage of this design strategy compared to single-well approaches is the ease of strain compensation due to the increased thickness of the (In,Ga)As.

\section{DESIGN STRATEGY}

Figure 1 depicts the conduction band edge $E_{c}$ and the calculated probability function for confined electronic states $^{14,15}$ in a representative DQW sample. The arrows on Fig. 1 indicate the intersubband transitions to be considered.

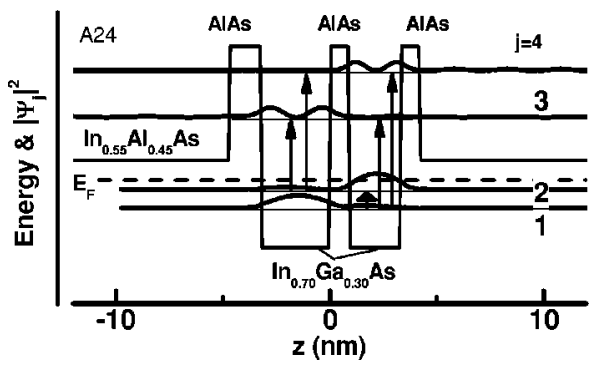

FIG. 1. Illustration of strain-compensated $\operatorname{In}_{0.70} \mathrm{Ga}_{0.30} \mathrm{As} / \mathrm{AlAs} /$ $\mathrm{In}_{0.55} \mathrm{Al}_{0.45} \mathrm{As} \mathrm{DQW}$ conduction-band profile $E_{c}$, with square moduli of the wave functions of $(j=1,2,3$, and 4$)$, states localized in $\operatorname{In}_{0.70} \mathrm{Ga}_{0.30} \mathrm{As}$. The dashed line represents the Fermi energy $E_{F}$. The arrows indicate the individual intersubband transitions to be considered. 


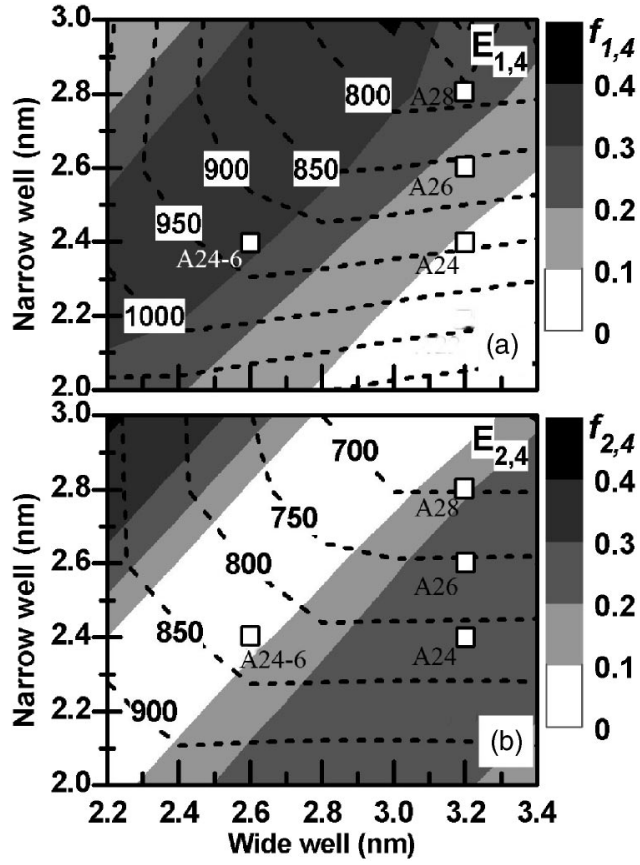

FIG. 2. Map of the oscillator strength (gray scale contours) and transition energies (dashed line contours) for intersubband transitions 1-4 [panel (a)] and 2-4 [panel (b)] over the field of the two well width parameters. Locations of the grown samples on the map are indicated by the open squares.

The highest-energy transitions are $2-4$ and $1-4$, the prime candidates for reaching the $1.55-\mu \mathrm{m}$ absorption band.

Besides controlling the absorption transition energy, the structure must also be designed to achieve an adequate $a b-$ sorption coefficient for the transition. The peak absorption coefficient per two-dimensional layer for a given transition at $T=0 \mathrm{~K}$ is given ${ }^{19}$ by

$$
a_{i, j}=\frac{n_{s} e^{2} \hbar}{2 \varepsilon_{0} c \eta m_{e} \Gamma} f_{i, j}
$$

where $n_{s}$ is a two-dimensional carrier density, $\eta$ is the refractive index, $m_{e}$ is an electron effective mass, $\Gamma$ is the line broadening, and $f_{i, j}$ is the oscillator strength ${ }^{20}$ for the transition.

Figure 2 illustrates the dependence of both oscillator strength and transition energy on the widths of the two quantum wells in the DQW structure, calculated within the effective-mass approximation. Either the 1-4 or the 2-4 transition can be optimized in terms of both transition energy and oscillator strength for certain well widths; the optimal ranges of well width are, however, very different for the two transitions: (i) Because the 1-4 transition takes place between energy levels primarily located in different wells, it is necessary that the wells be as similar as possible to promote mixing of the states between the wells in order to maximize the spatial overlap of the envelope wave functions. Thus the 1-4 transition is optimized for similar well widths, both small. (ii) On the other hand, because the 2-4 transition takes place between states both nominally located in the narrow well, the oscillator strength of the 2-4 transition is maximized with very different wide well and narrow well widths. (In a totally symmetric structure, including identical barriers, the 2-4
TABLE I. Sample parameters.

\begin{tabular}{ccccc}
\hline \hline No. & A28 & A26 & A24 & A24-6 \\
\hline$L_{\mathrm{NW}}(\mathrm{nm})$ & 2.8 & 2.6 & 2.4 & 2.4 \\
$L_{\mathrm{WW}}(\mathrm{nm})$ & 3.2 & 3.2 & 3.2 & 2.6 \\
$n_{\mathrm{QW}}\left(\mathrm{cm}^{-3}\right)$ & $1.1 \times 10^{19}$ & $1.1 \times 10^{19}$ & $1.1 \times 10^{19}$ & $0.9 \times 10^{19}$ \\
\hline \hline
\end{tabular}

transition is forbidden.) The optimized asymmetric design is for a very small narrow well width with a larger wide well width.

Thus, calculations show that either a symmetric or an asymmetric DQW design can lead to a high-energy intersubband transition with acceptable oscillator strength. The asymmetric design has an additional advantage that it allows more flexibility for strain balancing because the width of the thicker well is relatively unimportant in determining the transition energy. Therefore, using the asymmetric design described above, the 2-4 transition energy together with its oscillator strength can be controlled primarily based on the narrow well and the strain balancing through the wider well.

\section{EXPERIMENTAL AND DISCUSSION}

To investigate the effect of the widths of the two quantum wells on the transition energies and oscillator strengths for both the 1-4 and the 2-4 transitions, a series of structures was prepared in which these widths are individually varied.

The structures investigated here were grown using gassource molecular-beam epitaxy on a slightly compressively strained $\operatorname{In}_{0.55} \mathrm{Al}_{0.45} \mathrm{As}$ buffer on low-doped $n$-type InP:Sn or semi-insulating InP:Fe substrates. Typical growth conditions and characterization are described elsewhere. ${ }^{14}$

The heterostructures consist of 30 periods of AlAs- $\mathrm{In}_{0.70} \mathrm{Ga}_{0.30} \mathrm{As}-\mathrm{AlAs}-\mathrm{In}_{0.70} \mathrm{Ga}_{0.30} \mathrm{As}-\mathrm{AlAs}$ (1.5/ $\left.L_{\mathrm{WW}} / 0.9 / L_{\mathrm{NW}} / 0.9 \mathrm{~nm}\right) \mathrm{DQWs}$, each period separated by 15 $\mathrm{nm}$ of $\operatorname{In}_{0.55} \mathrm{Al}_{0.45} \mathrm{As}$. The widths of the narrower QW (NW) $L_{\mathrm{NW}}$, of the wider QW (WW) $L_{\mathrm{WW}}$, and doping level in the wells are listed for all samples in Table I. For all the samples doping is high enough that two lowest states, 1 and 2, are populated at $T=0 \mathrm{~K}$.

We first consider a sequence of structures with similar doping and the same $L_{\mathrm{WW}}$, but $L_{\mathrm{NW}}$ is varied. The midinfrared absorption in samples A28, A26, and A24 is depicted in Fig. 3. In all three samples, the energetic position of the strongest $1-3$ transition $(3.1 \mu \mathrm{m})$ is very similar because both states 1 and 3 are mostly localized in the $3.2-\mathrm{nm}$ well (WW). The primary effect of varying the narrower QW width $\left(L_{\mathrm{NW}}\right)$ is on the energies of states 2 and 4 because these states are mostly localized in the narrower well. Consequently, reducing the width of the narrower well causes a redshift of the 2-3 and a blueshift of 2-4 and 1-4 transitions. These trends are in broad agreement with the calculated transition energies, allowing a relatively reliable identification of the experimentally observed absorption peaks (Fig. 3). The energies of all experimentally observed transitions are, however, somewhat lower than their calculated values. This discrepancy can be attributed to possible grading of the heterointerface, ${ }^{14}$ slight deviation of the sample parameters 


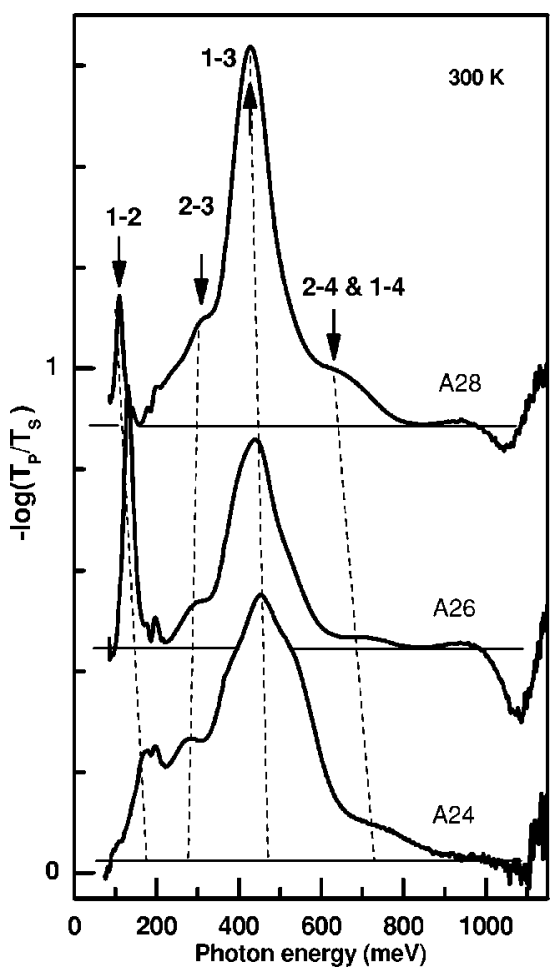

FIG. 3. Room-temperature intersubband absorption spectra of samples A24, A26, and A28. The same set of transitions, $1-2,2-3,1-3$, and $2-4$, is observed here for all the three samples. The observed energy shifts are consistent with the variation of the width of the narrow well. The vertical arrows indicate the individual absorption peaks. Transitions 2-4 and 1-4 are not resolved due to broadening. The three curves are shown with an offset for clarity, with the zero level for each curve indicated with the thin horizontal lines.

from the nominal values, as well as to the simplified treatment of the nonparabolicity of the conduction band in our calculations. $^{14,15}$

In the case of A24, the shoulder of the absorption band attributed to the overlaping 2-4 and 1-4 transitions extends beyond $800 \mathrm{meV}$.

Comparing the line shape of different absorption peaks (Fig. 3), we see that high-energy transitions are always broader than low-energy transitions. This difference is due to the subband dependence on the nonparabolicity of the energy dispersion $E_{c}^{i}(\mathbf{k})$. The absorption line broadening due to the combined non-parabolicity scales with subband separation. ${ }^{21}$ This effect is exacerbated in our samples because of the high doping level and its consequent range of populated states in $k$ space. Further, as noted above, the measured absorption peak energies are smaller than the calculated energy differences between the subbands involved in the transitions. This difference is about $10 \%$ for the 2-4 transitions and about $20 \%$ for the 1-4 transitions. Because subband 1 lies lower in energy than does subband 2, the Fermi momentum $k_{F}$ is larger. For this reason, the 1-4 transitions are expected to be broader than the 2-4 transitions and also, because of the nonparabolicity, to redshift the absorption peak from the calculated energy difference. The resulting broadening also tends to obscure high-energy transitions, even with large oscillator strength.

Figure 4 compares the room-temperature intersubband

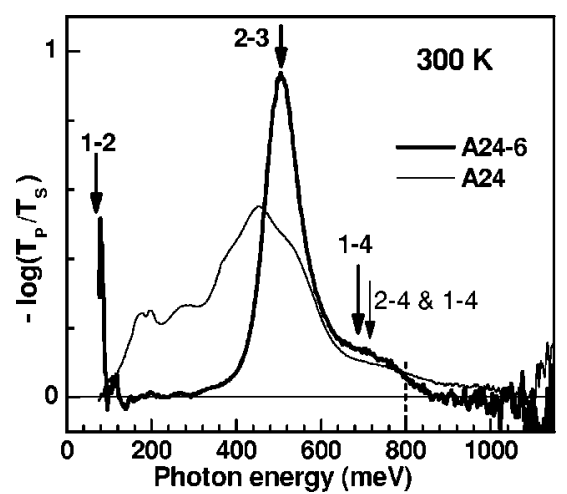

FIG. 4. Comparison of room-temperature intersubband absorption of sample A24-6 (thick line) and A24 (thin line). The vertical arrows (thick for A24-6 and thin for A24) indicate the individual absorption peaks.

absorption spectra of samples A24-6 and A24. Referring to Fig. 2 and Table I, samples A24 and A24-6 should be good candidates for achieving strong, short-wavelength absorptions based on the 2-4 and 1-4 transitions, respectively. Indeed, we do obtain relatively strong 2-4 and 1-4 absorption peaks, but centered at $730 \mathrm{meV}$ or $1.7 \mu \mathrm{m}$. Because the absorption peaks are relatively broad (as discussed above, for the same reason that they are redshifted), the absorption also extends to $800 \mathrm{meV}(1.55 \mu \mathrm{m})$. As discussed in Sec. II, both approaches-optimizing the 2-4 transition in the asymmetric sample A24 or optimizing the 1-4 transition in more symmetric sample A24-6-lead to comparable results in terms of achieving strong intersubband transitions with relatively high energy.

\section{CONCLUSIONS}

Summarizing, we describe a design strategy for shortwavelength intersubband absorption based on double quantum wells in the strain-compensated AlAs- $\mathrm{In}_{0.70} \mathrm{Ga}_{0.30} \mathrm{As}$ system and the conditions for short-wavelength absorption with large oscillator strength. Calculations indicate that for strong intersubband transitions based on the 1-4 transition, the narrow and wide wells should be similar in width, while for the 2-4 transitions, a greater difference in widths is necessary. The samples prepared using this design to optimize either the 1-4 or 2-4 transition strength are shown to have absorption peaks centered at $1.7 \mu \mathrm{m}$ and strong shortwavelength absorption out to $1.55 \mu \mathrm{m}$.

\section{ACKNOWLEDGMENTS}

The authors wish to acknowledge the support of the Deutsche Forschungsgemeinschaft within "Forschergruppe 394."

${ }^{1}$ R. Paiella, F. Capasso, C. Gmachl, C. G. Bethea, D. L. Sivco, J. N. Baillargeon, A. L. Hutchinson, and A. Y. Cho, Appl. Phys. Lett. 75, 2536 (1999).

${ }^{2}$ R. Paiella et al., Appl. Phys. Lett. 79, 2526 (2001).

${ }^{3}$ S. R. Schmidt, A. Seilmeier, and H. C. Liu, J. Appl. Phys. 91, 5545 (2002).

${ }^{4}$ S. Ehret, H. Schneider, J. Fleissner, P. Koidl, and G. Böhm, Appl. Phys. Lett. 71, 641 (1997).

${ }^{5}$ H. Schneider, C. Schönbein, G. Bihlmann, P. van Son, and H. Sigg, Appl. Phys. Lett. 70, 1602 (1997). 
${ }^{6}$ T. Akiyama, N. Georgiev, T. Mozume, H. Yoshida, A. V. Gopal, and O. Wada, IEEE Photonics Technol. Lett. 14, 495 (2002).

${ }^{7}$ R. Akimoto, Y. Kinpara, K. Akita, F. Sasaki, and S. Kobayashi Appl. Phys. Lett. 78, 580 (2001).

${ }^{8}$ R. Akimoto, K. Akita, F. Sasaki, and T. Hasama Appl. Phys. Lett. 81, 2998 (2002).

${ }^{9}$ C. Gmachl, H. M. Ng, S.-N. George Chu, and A. Y. Cho, Appl. Phys. Lett. 77, 3722 (2000).

${ }^{10}$ J. D. Heber, C. Gmachl, H. M. Ng, and A. Y. Cho, Appl. Phys. Lett. 81, 1237 (2002).

${ }^{11}$ A. Neogi, T. Mozume, H. Yoshida, and O. Wada, IEEE Photonics Technol. Lett. 11, 632 (1999).

${ }^{12}$ A. Guzman, J. Miguel-Sanchez, E. Luna, and E. Munoz, Proc. SPIE 5502 414 (2004)

${ }^{13}$ K. T. Lai, M. Missous, R. Gupta, and S. K. Haywood, J. Appl. Phys. 93 6063 (2003).

${ }^{14}$ N. Georgiev, T. Dekorsy, F. Eichhorn, M. Helm, M. P. Semtsiv, and W. T. Masselink, Appl. Phys. Lett. 83, 210 (2003), and references therein.
${ }^{15}$ M. P. Semtsiv, M. Ziegler, S. Dressler, W. T. Masselink, N. Georgiev, T. Dekorsy, and M. Helm, Appl. Phys. Lett. 85, 1478 (2004).

${ }^{16}$ M. Missous, C. Mitchell, J. Sly, K. T. Lai, R. Gupta, and S. K. Haywood, Physica E (Amsterdam) 20, 496 (2004)

${ }^{17}$ Y. Hirayama, J. H. Smet, L. H. Peng, C. G. Fonstad, and E. P. Ippen, Appl. Phys. Lett. 63, 1663 (1993).

${ }^{18}$ J. H. Smet, L. H. Peng, Y. Hirayama, and C. G. Fonstad, Appl. Phys. Lett. 64, 986 (1994).

${ }^{19}$ M. Helm, Semicond. Sci. Technol. 10, 557 (1995).

${ }^{20}$ The oscillator strength is calculated using expression $f_{1,2}$ $=\frac{2 E_{i, j}}{\hbar^{2}}\left|\oint \Psi_{i}(z) \sqrt{m_{e}(z)} \cdot z \cdot \psi_{j}(z) d z\right|^{2}$, where $m_{e}(z)$ is an electron effective mass taken to be $m_{e}^{\text {InGaAs }}=0.035 \times m_{0}$ for $\operatorname{In}_{0.70} \mathrm{Ga}_{0.30} \mathrm{As}, m_{e}^{\mathrm{AlAs}}=0.115 \times m_{0}$ for AlAs, and $m_{e}^{\text {InAlAs }}=0.075 \times m_{0}$ for $\operatorname{In}_{0.55} \mathrm{Al}_{0.45} \mathrm{As} . \psi_{i}(z)$ and $\psi_{j}(z)$ are the envelope wave functions in $z$ direction of states $i$ and $j$ correspondently. $E_{i, j}$ is the energy spacing of states $i$ and $j$.

${ }^{21}$ U. Ekenberg, Phys. Rev. B 36, 6152 (1987). 